\title{
Piezoresponse force microscopy of lead titanate nanograins possibly reaching the limit of ferroelectricity
}

\author{
A. Roelofs ${ }^{\mathrm{a})}$ and T. Schneller \\ Institut für Werkstoffe der Elektrotechnik, RWTH Aachen, D-52056 Aachen, Germany \\ K. Szot and R. Waser \\ Elektrokeramische Materialen, Institut für Festkörperforschung, Forschungszentrum Jülich, \\ D-52425 Jülich, Germany
}

(Received 14 May 2002; accepted 9 November 2002)

\begin{abstract}
Single ferroelectric lead titanate (PTO) grains down to $15 \mathrm{~nm}$ were fabricated by chemical solution deposition. Varying the dilution of the precursor solution leads to different grain sizes between 15 and $200 \mathrm{~nm}$. The grain-size-dependent domain configuration was studied using three-dimensional piezoresponse force microscopy (PFM). It is found that the PTO grains in a dense film contain laminar $90^{\circ}$ domain walls, whereas separated PTO grains show more complicated structures of mainly $180^{\circ}$ domain walls. For grains smaller than $20 \mathrm{~nm}$, no piezoresponse was observed and we suppose this could be due to the transition from the ferroelectric to the superparaelectric phase which has no spontaneous polarization. Recent calculations showed that the ferroelectricity of fine ferroelectric particles decrease with decreasing particle size. From these experiments the extrapolated critical size of PTO particles was found to be around 4-14 nm. (C) 2002 American Institute of Physics. [DOI: 10.1063/1.1534412]
\end{abstract}

The increasing interest in ferroelectric thin films for ferroelectric-random-access-memories applications leads to the question: how small can a ferroelectric capacitor be and still have a stable switchable remanent polarization? Until now there are only some calculations predicting a transition from the ferroelectric phase to a superparaelectric phase if the cell size (here grain size) becomes small enough. ${ }^{1,2}$ [The estimated transition size for lead titanate (PTO), finite in three dimensions, calculated by Wang et al. is $7.4 \mathrm{~nm}]^{3}$ Most experimental studies of size effects use a top-down approach starting with a dense ferroelectric thin film and then use electron-beam or focused-ion-beam methods to structure (lateral) small devices. ${ }^{4,5}$ With the top-down approach it is difficult to distinguish between the real intrinsic size effects and effects induced by the processing steps. Another way is by studying nanopowder of the material ${ }^{2}$ but the data are limited to $\mathrm{x}$ ray and transelectron microscopy data.

In this work we apply a bottom-up approach. A chemical solution deposition (CSD) method for the preparation of $\mathrm{PTO}^{6}$ thin films is modified to achieve separated single PTO grains in the size ranging from 200 to less than $20 \mathrm{~nm}$ (unpublished). This method opens up the way to study intrinsic size effects of several ferroelectric materials using an easy deposition method without the need of sophisticated structuring methods.

To investigate nanoscale domain mapping and switching, piezoresponse force microscopy ${ }^{7-9}$ is applied. Topological and ferroelectric properties of individual grains along all three dimensions are monitored. In contrast to conventional macroscopic electrical characterization using extended top electrodes, piezoresponse force microscopy (PFM) may be used to study ferroelectric thin films in the early growth

a)Electronic mail: roelofs@iwe.rwth-aachen.de stage having a not complete coverage. Whereas extended top electrodes would be shorted making macroscopical electrical characterization impossible. PFM may be addressed both for inspection and manipulation of single grains.

The PTO nanograins were deposited onto $\mathrm{Si} / \mathrm{SiO}_{2} / \mathrm{TiO}_{2} / \mathrm{Pt}$ substrates. An anhydrous lead acetate based precursor solution ${ }^{10,11}$ was spin coated onto the substrate and dried for $2 \mathrm{~min}$ on a hot plate at $350^{\circ} \mathrm{C}$. Crystallization was initiated by a rapid thermal annealing process at $700{ }^{\circ} \mathrm{C}$ for $10 \mathrm{~min}$, resulting in separated single PTO grains. The separated PTO grains have no predominant crystallographic orientation as proven by $\mathrm{X}$-ray diffraction experiments. Figure 1 shows a scanning electron microscope image of a sample with grain sizes in the range of 15-200 nm.

Nanoscale inspection of the ferroelectric grains was carried out using a modified scanning force microscope ${ }^{12}$ from JEOL $^{\mathrm{TM}}$. Comparable experimental setups can be found elsewhere. ${ }^{13-15}$ Operation in the PFM mode allows simultaneous recording of three different information: sample topography, out-of-plane polarization (OPP), and in-planepolarization (IPP) along the $x$-scan direction (via cantilever torsion).

In Fig. 2 the piezoresponse image of a relatively dense PTO film is shown. The domain configuration in the different grains mostly show simple equidistant stripes of width ranging from 12 to about $80 \mathrm{~nm}$. Analyzing the IPP image and the OPP image simultaneously show that these stripes are representing $90^{\circ}$ domains. For example when we look within the circle in Fig. 2(a) (OPP image) it shows, black and gray stripes in the grain. The black region indicates the projection of the polarization onto the $z$ axis which points out of the plane of the paper. The gray stripes demonstrate that the polarization in these regions is lying in the $x-y$ plane and therefore not leading to any piezoresponse signal in the $z$ direction. (This information is enough to know that these 


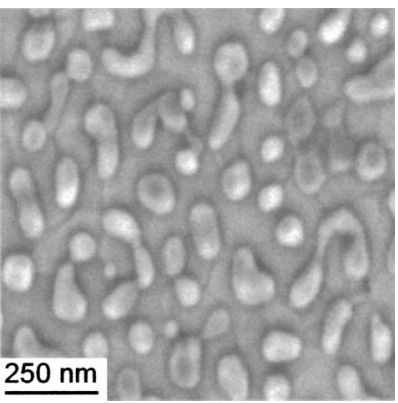

FIG. 1. Scanning electron microscope image of PTO nanograins deposited onto a $\mathrm{Si} / \mathrm{SiO}_{2} / \mathrm{TiO}_{2} / \mathrm{Pt}$ substrate using a modified CSD route. Grains of different shape and sizes can be found. The grain sizes range from about 100 $\mathrm{nm}$ down to several $10 \mathrm{~nm}$.

neighboring domains form $90^{\circ}$ domain walls.) Taking into account that the domains will have a "head-to-tail" configuration (for noncharged domain walls) leads to the conclusion that the polarization in the gray stripes of this grain will point into the negative $y$ direction. This analysis can be done for each grain and it is found that these stripes indicate domains with $90^{\circ}$ domain walls. For the line scan over the grain marked with an arrow in the IPP image, Fig. 2(b) shows the excellent resolution of the measurement. Domains with a width of $12 \mathrm{~nm}$ can clearly by distinguished. We believe that these $90^{\circ}$ domain walls are caused due to the mechanical stress that the ferroelectric grains exert onto each other, and not due to the stress caused by the different thermal expansion of the substrate and the PTO film. Because, having a look at PTO grains obtained from higher diluted solution, that are separated from each other (no image shown) show a more complicated domain configuration. Analyzing the data as done earlier leads to the conclusion that the separated grains mostly exhibit a domain configuration with $180^{\circ}$ domain walls. As the stress between the substrate and each grain will be the same for a dense PTO film and a film consisting of separated grains, the difference in the domain configuration can only be explained by the additional stress the grains cause among themselves.

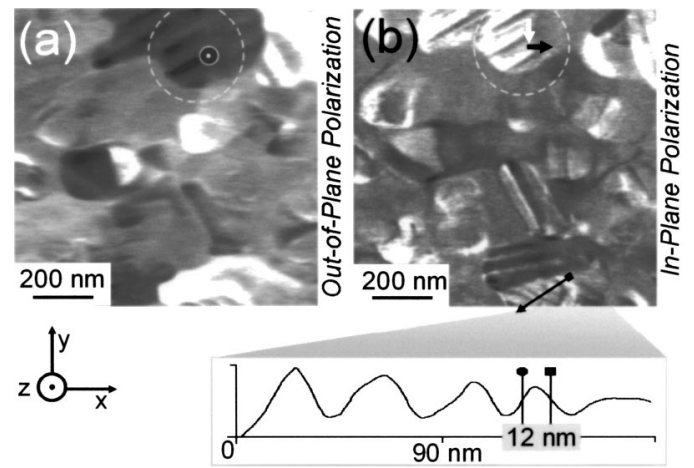

FIG. 2. Piezoresponse image of a dense PTO film prepared by a standard CSD method with a nondiluted precurser solution. In (a) the out-of-plane polarization (OPP) is shown. The grain in the upper part of the image marked with a circle consists of $90^{\circ}$ domains. Black indicates that the projection of the polarization onto the $z$ axis is pointing into the positive $z$ direction. Black in the in-plane image (b) signifies that the projection of the polarization onto the $x$ axis points into the negative $x$ direction. In the lower part of the image (b) a line scan over the grain marked with an arrow exhibits the excellent resolution of the PFM, clearly showing domains with

they do not have any permanent polarization.
Downloaded 21 Dec 2006 to 134.94.122.39. Redistribution subject to AIP license or copyright, see http://apl.

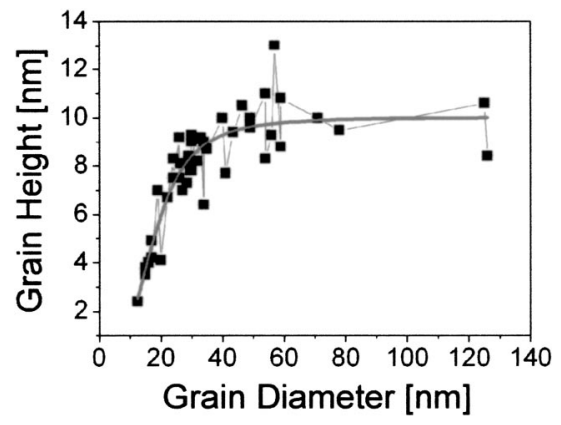

FIG. 3. Grain height $\left(G_{\text {height }}\right)$ as a function of grain diameter $\left(G_{\text {diam. }}\right)$. The solid line is the fitted Hill function $G_{\text {height }}=G_{\text {max }} \cdot G_{\text {diam. }}^{n} /\left(k^{n}+G_{\text {diam }}^{n}\right)$. The maximum grain height $\left(G_{\max }\right)$ is $10 \mathrm{~nm}, n$ is about 4 and $k$ is approximately 17. For PTO islands larger than $40 \mathrm{~nm}$ the average height is $10 \mathrm{~nm}$, smaller islands are lower down to $2.4 \mathrm{~nm}$ for a grain diameter of $12 \mathrm{~nm}$.

When we look at the size and height distribution of the separated PTO grains it is noticed that the grains larger than $50 \mathrm{~nm}$ have an average height of $10 \mathrm{~nm}$ (Fig. 3). Smaller grains have a decreasing height. The smallest measured grain had a diameter of about $12 \mathrm{~nm}$ and the height was $2.4 \mathrm{~nm}$. The average grain density was 120 PTO grains per square micron.

Most fascinating is the finding represented in Fig. 4. The topographic image clearly shows 11 PTO grains of which one (marked with the white circle) does not lead to a piezoresponse signal as shown in the piezoresponse images Figs. 4(b) and 4(c). The grain size can be estimated from the line scan to be $20 \mathrm{~nm}$. We believe that at this grain size the transition from the ferroelectric to the superparaelectric phase could take place. Although Tybell et al. ${ }^{16}$ reported on PFM measurements on epitaxial thin films down to $4 \mathrm{~nm}$, this seems to be contradicting our results. This could be explained either by the different stress induced through the different substrate they are using, making the ferroelectric phase stable for thinner films or due to the fact that they shrink the ferroelectric only in one dimension and not in all

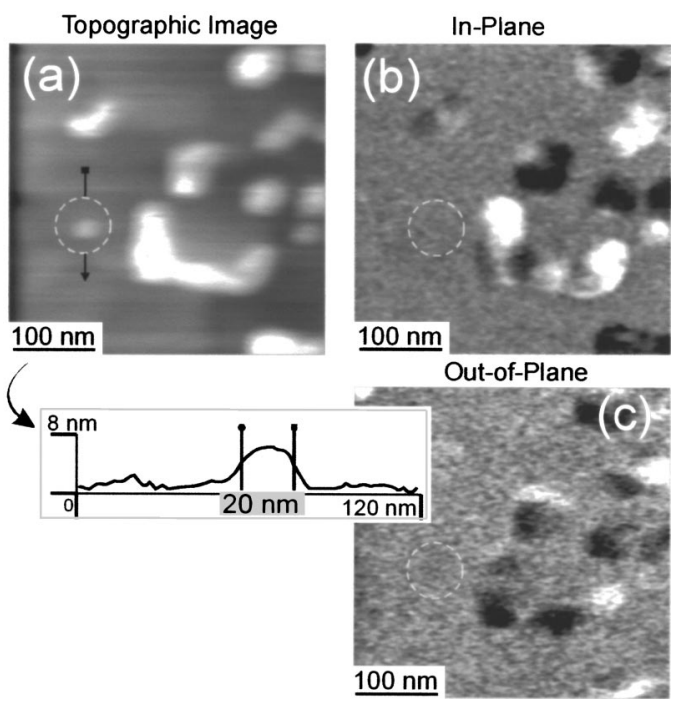

FIG. 4. The topographic image (a) shows 11 PTO grains of sizes between 100 down to $20 \mathrm{~nm}$ indicated by the circles. In the line scan over the grain denoted with an arrow, shown at the bottom, the size of the grains can be determined. In the PFM images (b) in-plane and (c) out-of-plane piezoresponse the grain of the size of $20 \mathrm{~nm}$ is not visible leading to the assumption they do not have any permanent polarization. 
three as in this approach. Ferroelectricity is viewed as a collective phenomenon with a spontaneous polarization resulting from the alignment of localized dipoles within a correlation volume, ${ }^{17}$ hence, it is important to shrink all three dimensions.

Theoretical studies ${ }^{1}$ predict a phase transition for PTO from the tetragonal phase (ferroelectric phase) to a cubic (nonferroelectric) phase, so called superparaelectric phase if the size of a PTO cell becomes smaller than several nanometers. Furthermore, it can be seen that the domain configuration for single ferroelectric grains become more simple with the reduction in grain size. For grains in the range of about $40-50 \mathrm{~nm}$ we often find only two domains, but if the grain size becomes smaller than $40 \mathrm{~nm}$ we find only monodomain grains. Scanning the sample in contact mode and applying a dc voltage between the platinum bottom electrode and the conductive cantilever monitoring the local electrical current enabled us to ensure that even the smallest grains that do not show piezoresponse are at least dielectric. This rules out the possibility of these grains being platinum hillocks.

In conclusion, we presented a simple bottom up approach to achieve separated ferroelectric PTO grains down to $20 \mathrm{~nm}$. It was found that the domain configuration was strongly dependent on the size of the grains. For dense PTO films the grains are dominated by $90^{\circ}$ domain walls whereas $180^{\circ}$ domain walls are governing the separated grains. Grains that were smaller than $20 \mathrm{~nm}$ did not show any piezoresponse that led us to the conclusion that this could be the limiting size for the ferroelectric phase.

${ }^{1}$ W. L. Zhong, Y. G. Wang, P. L. Zhang, and B. D. Qu, Phys. Rev. B 50, 698 (1994); C. L. Wang and S. P. Smith, J. Phys.: Condens. Matter 7, 7163 (1995); S. Li, J. A. Eastman, J. M. Vetrone, C. M. Foster, R. E. Newnham, and L. E. Cross, Jpn. J. Appl. Phys., Part 1 36, 5169 (1997).
${ }^{2}$ B. Jiang, J. L. Peng, W. L. Zhong, and L. A. Bursill, J. Appl. Phys. 87, 3462 (2000); W. L. Zhong, B. Jiang, P. L. Zhang, J. M. Ma, H. M. Cheng, and Z. H. Yang, J. Phys.: Condens. Matter 5, 2619 (1993); K. Ishikawa, K. Ishikawa, T. Nomura, N. Okada, and K. Takada, Jpn. J. Appl. Phys., Part 1 35, 5196 (1996); S. Chattopadhaya, P. Ayyub, V. R. Palkar, and M. Multani, Phys. Rev. B 52, 13177 (1995).

${ }^{3}$ Y. G. Wang, W. L. Zhong, and P. L. Zhang, Phys. Rev. B 51, 17235 (1995).

${ }^{4}$ C. S. Ganpule, A. Stanishevsky, S. Aggarwall, J. Melengailis, E. Williams, and R. Ramesh, Appl. Phys. Lett. 75, 3874 (1999).

${ }^{5}$ M. Alexe, C. Harnagea, W. Erfurth, D. Hesse, and U. Goesele, Appl. Phys. A: Mater. Sci. Process. 70, 247 (2000).

${ }^{6}$ D. Bolten, M. Hoffmann, U. Hasenkox, O. Lohse, and R. Waser, Ferroelectrics 225, 117 (1999).

${ }^{7}$ L. M. Eng, H. J. Güntherodt, G. A. Schneider, U. Köpke, and J. Muñoz Saldaña, Appl. Phys. Lett. 74, 233 (1999).

${ }^{8}$ A. Roelofs, F. Schlaphof, S. Trogisch, U. Böttger, R. Waser, and L. M. Eng, Appl. Phys. Lett. 77, 3444 (2000).

${ }^{9}$ A. Roelofs, N. A. Pertsev, R. Waser, F. Schlaphof, L. M. Eng, C. Ganpule, V. Nagarajan, and R. Ramesh, Appl. Phys. Lett. 80, 1424 (2002).

${ }^{10}$ R. Nouwen, J. Mullens, D. Franco, J. Yperman, and L. C. Van Poucke, Vib. Spectrosc. 10, 291 (1996).

${ }^{11}$ D. J. Wouters, G. J. Norga, and H. E. Maes, Mater. Res. Soc. Symp. Proc. 541, 381 (1999).

${ }^{12}$ G. Binnig and H. Rohrer, Helv. Phys. Acta 55, 726 (1982).

${ }^{13}$ K. Franke, J. Besold, W. Haessler, and C. Seegebarth, Surf. Sci. Lett. 302, L283 (1994); T. Hidaka, T. Maruyama, I. Sakai, M. Saitoh, L. A. Wills, R. Hiskes, S. A. Dicarolis, J. Amano, and C. M. Foster, Integr. Ferroelectr. 17, 319 (1997); O. Auciello, A. Gruverman, H. Tokumoto, A. S. Prakash, S. Aggarwal, and R. Ramesh, MRS Bull. 23, 33 (1998); M. Abplanalp, L. M. Eng, and P. Günter, Appl. Phys. A: Mater. Sci. Process. 66A, S231 (1998).

${ }^{14}$ A. Gruverman, O. Auciello, and H. Tokumoto, Annu. Rev. Mater. Sci. 28, 101 (1998); A. Gruverman, A. Kholkin, A. Kingon, and H. Tokumoto, Appl. Phys. Lett. 78, 2751 (2001).

${ }^{15}$ C. S. Ganpule, V. Nagarajan, H. Li, A. S. Ogale, D. E. Steinhauer, S. Aggarwall, E. Williams, and R. Ramesh, Appl. Phys. Lett. 77, 292 (2000).

${ }^{16}$ T. Tybell, C. H. Ahn, and J. M. Triscone, Appl. Phys. Lett. 75, 856 (1999).

${ }^{17}$ M. E. Lines and A. M. Glass, Principles and Applications of Ferroelectrics and Related Materials (Oxford University Press, Oxford, 1977). 